\title{
Short Communication: In Vitro Accumulation of Drug Resistance Mutations in Chimeric Infectious Clones Containing Subtype B or C Reverse Transcriptase and Selected with Tenofovir or Didanosine
}

\author{
Rodrigo D. Cunha, Celina M. Abreu, Arielly K.P. Sousa, Viviana C.J. Mabombo, \\ Monique Nijhuis, ${ }^{2}$ Dorien de Jong, ${ }^{2}$ and Amilcar Tanuri ${ }^{1}$
}

\begin{abstract}
Highly active antiretroviral therapy (HAART) contributed to the improvement in the life expectancy of HIVinfected patients. However, the emergence of drug-resistant mutations (DRM) is a major viral factor impacting therapeutic failure. Differences in DRM can occur among HIV-1 subtypes. We evaluate the kinetics of the selection of resistance mutations in vitro analyzing two chimeric clones that contain the reverse transcriptases of subtypes B or $\mathrm{C}\left(\mathrm{RTB}^{\prime}\right.$ and $\left.\mathrm{RTC}^{\prime}\right)$ in cells treated with increasing concentrations of tenofovir disoproxil fumarate (TDF) and didanosine (ddI). The mutation K65R is selected more quickly in $\mathrm{RTC}^{\prime}$ than in $\mathrm{RTB}^{\prime}$ viruses with TDF and ddI, and additional mutations (positions 45, 62, and 68) were selected after K65R fixation. Other primary mutations (M184V and Q151M) were selected with ddI treatment in conjunction with K65R only in RTC' viruses. Both patterns, M184V + K65R and Q151M + K65R, have a significant impact on NRTI resistance. Our data suggest that selection of TDF and ddI DRMs can occur earlier in subtype C HIV in patients when compared to subtype B.
\end{abstract}

A BOUT 35.3 MILLION PEOPLE were living with HIV-1 in 2012 worldwide with the majority of them living in subSaharan Africa. Highly active antiretroviral therapy (HAART) reduced the number of HIV-1-infected people and HIV-related deaths per year. ${ }^{1}$ This regimen is composed of two nucleoside analogue reverse transcriptase (RT) inhibitors (NRTIs) and one nonnucleoside analogue RT inhibitor (NNRTI) or a protease inhibitor (PI) as the first line of treatment. PIs are not used as first-line treatment in low- to middle-income countries and are reserved for second-line regimens. Conversely, NRTIs are used worldwide, although the drugs of choice depend on the resources of the country.

Tenofovir disoproxil fumarate (TDF) and emtricitabine (FTC) are widely used in high-resource countries. Zidovudine (AZT), lamivudine (3TC), and stavudine (d4T) are chosen in low-resource countries and are recommended by the World Health Organization. ${ }^{2}$ Didanosine (ddI) is no longer used in most countries, although it is one of the few options available in some African and Asian countries. Moreover, ddI is still administered in children and is recommended as one of the second-line medications in South Africa. ${ }^{3-5}$ Integrase and entry inhibitors, when available, are used in salvage regimens whenever second-line therapeutic failure is observed, confirmed by an increase in viral load and/or a decrease in CD4 measurements. ${ }^{2}$ The large-scale implementation of HAART in countries with limited resources improved the quality of life and the life expectancy of HIV-1-infected patients, ${ }^{6}$ but drug resistance mutations (DRM) still represent an important factor involved in therapeutic failure.

HIV-1 is classified into many types, subtypes, and circulating recombinant forms. The epidemic in developed countries is dominated by subtype $\mathrm{B}$, which is thus the target of most studies. Nevertheless, subtype $\mathrm{C}$ is responsible for the majority of HIV-1 infections worldwide due to its high prevalence in sub-Saharan Africa. Moreover, this variant is also found in India, China, and Brazil. ${ }^{7}$

Genetic differences among HIV-1 subtypes are around $10 \%$ in the pol gene. Therefore, it could be predicted that different resistance mutations or different mutation patterns could emerge in different HIV-1 subtypes under the same antiretroviral pressure. ${ }^{8}$ In fact, differences in DRM position, amino acid composition, and time of acquisition were observed during HAART among HIV-1 subtypes. Differences

\footnotetext{
${ }^{1}$ Laboratório de Virologia Molecular, Universidade Federal do Rio de Janeiro, Rio de Janeiro, Rio de Janeiro, Brazil.

${ }^{2}$ Department of Virology, Medical Microbiology, University Medical Center Utrecht, Utrecht, the Netherlands.
} 
in the prevalence of resistance mutations at codon 106 that were selected by NNRTIs were observed between subtype B and $\mathrm{C}$ viruses. In this case, V106A accumulated in subtype $\mathrm{B}$ viruses treated with nevirapine while V106M emerged in subtype $\mathrm{C}$ viruses under efavirenz treatment. These events can be explained by differences in codon usage at that position. ${ }^{9}$ In phenotypic assays, V106M confers resistance to efavirenz (EFV) and nevirapine (NVP) while V106A impacts only NVP. ${ }^{10}$

Recently, our group has shown that differences in thymidine analogue mutation (TAM) pathways during AZT in vitro selection were observed between chimeric viruses generated with RTs from subtypes B and C, with the TAM-1 pathway (T215Y) being selected in subtype B and the TAM-2 (T215F, K70R) pathway being selected in subtype $C$ viruses. In contrast, no differences at selected DRMs were observed with 3TC treatment between these two subtypes. ${ }^{11}$ It is acknowledged, however, that TAM-1 has a higher impact in resistance to AZT and a large impact in cross-resistance. ${ }^{12}$

The K65R mutation, which confers resistance to all NRTIs except AZT, was reported to occur with low frequency in patients infected with subtype B. However, this DRM has been found to occur with higher frequency in subtype $\mathrm{C}$ viruses. ${ }^{13}$ In addition, K65R is selected more quickly in subtype $\mathrm{C}$ viruses when compared to subtype $\mathrm{B}$ viruses when subjected to the selective pressure of TDF in vitro. ${ }^{14}$ Nevertheless, preliminary data on clinical assays with TDF treatment somehow showed discordant results. ${ }^{15}$

Here we evaluated the acquisition kinetics of resistance mutations when chimeric infectious clones containing RTs from subtype $\mathrm{B}$ or $\mathrm{C}$ were subjected to in vitro selection with TDF or ddI, which is another NRTI known to select the K65R mutation in resistant viruses.

Different fragments of the wild-type (WT) subtype B and C RT sequence were cloned into molecular clones pHXB2 $\triangle \mathrm{RT}$ and $\mathrm{pHXB} 2 \Delta \mathrm{NRT}$, respectively. The first plasmid $\mathrm{pHXB} 2 \Delta \mathrm{RT}$ has the almost complete genome of HXB2 except for the RT gene, which was deleted in codons 25 to 554 and unique sites of restriction enzymes NgoMIV and MluNI were inserted through an adapter. We amplified NL4-3 RT, a subtype B clone, with primers RT2569 and RT22 in the first round of the polymerase chain reaction (PCR) $(1,744 \mathrm{bp})$ followed by RTball and NgoMIV-INT1rev in the second round of the PCR $(1,623 \mathrm{bp}){ }^{16}$ Therefore, the PCR product will contain the same flanking restriction enzyme sites as the molecular clone and both of them were digested and ligated to generate HXB2 RTpNL $4.3\left(\mathrm{RT}_{\mathrm{B}^{\prime}}\right)$.

The second plasmid, $\mathrm{pHXB} 2 \Delta \mathrm{NRT}$, also contains the HXB2 genome and the RT N-terminal region of the connection domain (codons 25 to 315 ) was deleted and two distinct restriction enzyme sites were inserted for cloning purposes (MluNI and Van91I). For subtype C RT cloning, a sample from a naive patient from the south of Brazil (C23) was amplified with primers RT2569 and 3'RTAA339 in the first round PCR (967 bp) and RTball and RT21 in the second round PCR (941 bp) as previously described. ${ }^{17}$ To clone the PCR fragment we used the same strategy explained above to generate CNRT23 $\left(\mathrm{RT}_{\mathrm{C}^{\prime}}\right)$.

Several differences could be observed between RTB' and RTC $^{\prime}$ in amino acid sequences (V35T, E36A, T39D, K43R, S48T, Q102K, D121Y, K122E, C162S, K173N, Q174K, D177E, T200A, Q207E, and R211K) since each subtype has a different polymorphic signature in the RT region. However, none has previously been associated with ddI or TDF resistance. All viral stocks were titrated through tissue culture infectious dose $50 \%\left(\right.$ TCID $\left._{50}\right)$ in MT4 cells before being used in selection experiments.

MT4 cells were infected with chimeric virus RTB' or RTC' in six independent infections (technical replicates) at a multiplicity of infection (MOI) of 0.002 to minimize stochastic effects at drug resistance mutation selection. These viruses were subjected to serial passage with increasing concentrations of TDF and ddI for 142 and 96 days, respectively. The start concentration was $10 \%$ of the half-maximal effective concentration $\left(\mathrm{EC}_{50}\right)$ (Fig. 1). If low levels of infection were detected by optical microscopy through cell death and/or characteristic cytopathic effect evaluation, the antiretroviral (ARV) concentration was maintained for the next passage. The ARV concentration was doubled if higher levels of infection were obtained in the previous passage. After each passage, viruses present in supernatants were subjected to genomic RNA extraction and RT gene amplification with specific primers. ${ }^{11}$ Amplicons were sequenced by the Sanger method and manually analyzed (two different persons) for the presence of drug resistance mutations within the RT gene. When two peaks (WT nucleotide and mutate nucleotide) were observed, we concluded that a mixture existed and determined a partial selection. However, when only a peak of a nucleotide different from the WT was observed, we concluded that this mutation was fixed.

Replicative capacity analyses of viruses selected with TDF were performed in MT4 cells. These cells were infected with $5 \mathrm{ng}$ of p24 for each virus in triplicate assays. RTB' WT and $\mathrm{RTC}^{\prime}$ WT infections in the absence of TDF and RTB ${ }^{\prime}$ carrying K65R, K65R + A62T, and K65R + G45R, as well RTC' carrying K65R, K65R + S68S/N, and K65R + S68S/G in the presence of $0.2 \mu \mathrm{M}$ TDF. Supernatants from infections were collected and analyzed for p24 levels at days 1, 3, and 5 after infection using Retro-Tek HIV-1 p24 antigen ELISA (Zeptometrix) as described by the manufacturer.

Analysis of TDF and ddI selection revealed differences as drug concentrations were increased over time (Fig. 1A and B). The TDF concentration was frequently doubled every passage. In addition, a similar pattern was observed for six $\mathrm{RTB}^{\prime}$ and RTC' independent infections (Fig. 1A). In contrast, the ddI concentration reached a plateau and concentration enhancement was very difficult due to the cytotoxic effects caused by the drug. Differences between RTB' and RTC' infections could be observed only at the last passages (Fig. 1B). The fact that $\mathrm{RTB}^{\prime}$ and $\mathrm{RTC}^{\prime}$ viruses have a similar pattern of drug concentration increase suggests that the replicative capacity of $\mathrm{RTB}^{\prime}$ and $\mathrm{RTC}^{\prime}$ viruses is similar.

To evaluate this MT4 cells were infected with RTB' and $\mathrm{RTC}^{\prime}$ viruses in triplicate in the absence of drugs and were followed for 5 days. Supernatant was collected and analyzed at days 1, 3, and 5 for p24 levels. We can see that RTB' p24 levels were higher at day 3 while $\mathrm{RTC}^{\prime}$ levels were higher only at day 5 (Fig. 1C) implying that $\mathrm{RTB}^{\prime}$ viruses have a higher replicative capacity than $\mathrm{RTC}^{\prime}$ viruses. These data are not surprising as subtype B background chimeras carrying subtype C RT usually have lower replicative capacity. ${ }^{18}$

The $\mathrm{EC}_{50}$ value for $\mathrm{ddI}$ is 1,000 times higher than TDF. Thus, the final concentration of ddI at in vitro selection can reach only $0.1-1 \mathrm{mM}$, about 5-50 times the $\mathrm{EC}_{50}$ for $\mathrm{ddI}$. 


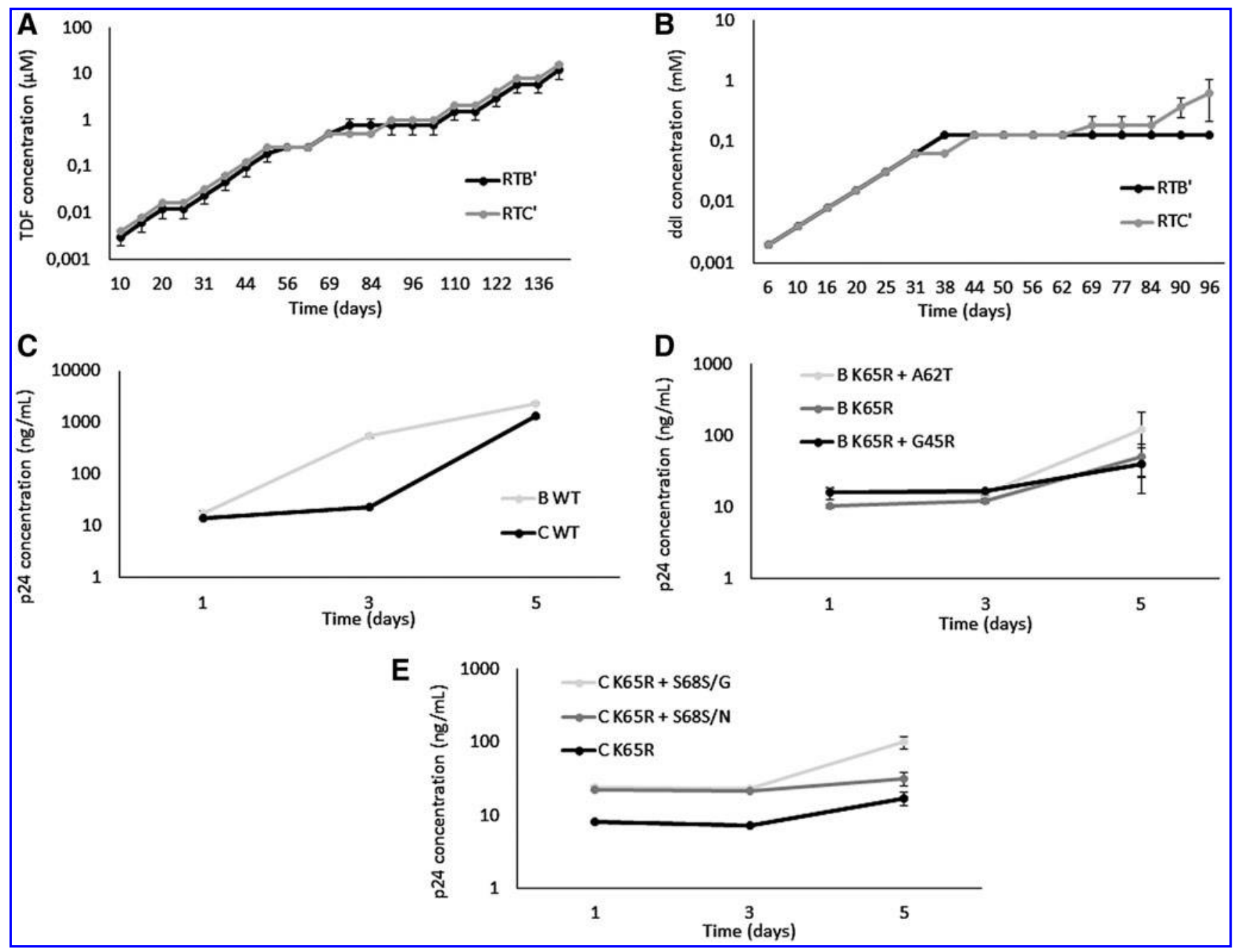

FIG. 1. Enhancement of drug concentrations at in vitro selections and replicative capacity analysis. Increase at tenofovir disoproxil fumarate (TDF) (A) and didanosine (ddI) (B) concentrations along in vitro selection. When HIV infection reaches higher levels of cell death and cytopathic effect at previous passage, the drug concentration was doubled. However, when low levels of each were observed, the drug concentration was maintained at the next passage. The average concentration of six independent infections for the reverse transcriptases of subtypes B and C (RTB' and $\mathrm{RTC}^{\prime}$ ) and standard deviation values are shown over time. Viruses $\mathrm{RTB}^{\prime}$ and $\mathrm{RTC}^{\prime} \mathrm{WT}(\mathbf{C})$ or viruses carrying resistance mutations $(\mathbf{D}, \mathbf{E})$ were used to infect MT-4 cells for 5 days in the absence or $0.2 \mu \mathrm{M}$ TDF, respectively. Supernatant was collected and p24 levels were analyzed on days 1,3 , and 5 .

Conversely, the final concentration of TDF can be increased to $8-16 \mu \mathrm{M}$, about 400 - to 800 -fold of the $\mathrm{EC}_{50}$ for TDF.

Our data demonstrate that $\mathrm{K} 65 \mathrm{R}$ is the major mutation selected with TDF treatment, corroborating the literature. ${ }^{19}$ Interestingly, this mutation was both partially and completely selected faster in $\mathrm{RTC}^{\prime}$ virus when compared to $\mathrm{RTB}^{\prime}$ virus in all six replicates (Table 1 ). It was partially detected at 31-50 days (median: 47 days) of in vitro selection in $\mathrm{RTC}^{\prime}$ viruses versus 62-84 days (median: 77 days) in RTB $^{\prime}$ viruses and completely emerged at 50-56 days (median: 53 days) in RTC' viruses versus 84-90 days (median: 90 days) in RTB' viruses. These results could not be explained by differences in replicative capacity since $\mathrm{RTC}^{\prime}$ viruses have a replicative capacity lower than RTB' viruses (Fig. 1C).

Previous studies found results comparable to the results presented here. However, viruses used in these experiments were isolated from peripheral blood mononuclear cells (PBMCs) from HIV-infected patients. This methodological approach has limitations since isolated viruses were not clonal and mutations could already be present in minority populations. ${ }^{14}$ The viruses used in our experiments were clonal and corroborate the results from Brenner et al. ${ }^{14}$ However, other studies have shown that there is no difference in required time to accumulate $\mathrm{K} 65 \mathrm{R}$ in subtype $\mathrm{B}$ or $\mathrm{C}$ when samples from patients under a HAART-containing TDF regime were used. ${ }^{15}$

In fact, differences in treatment regimen were observed in those studies. When TDF monotherapy is used, as in our study, K65R is selected faster in subtype C when compared to subtype B recombinant viruses. ${ }^{14}$ Conversely, no differences in K65R accumulation time between RT subtypes B and C were observed when TDF was used as part of a triple therapy HAART ${ }^{15}$ The impact of TDF could be lower as other ARVs also would affect the selection of resistance viruses when HAART was utilized. Nonetheless, Sunpath et al. demonstrated that K65R was selected at a higher frequency in 


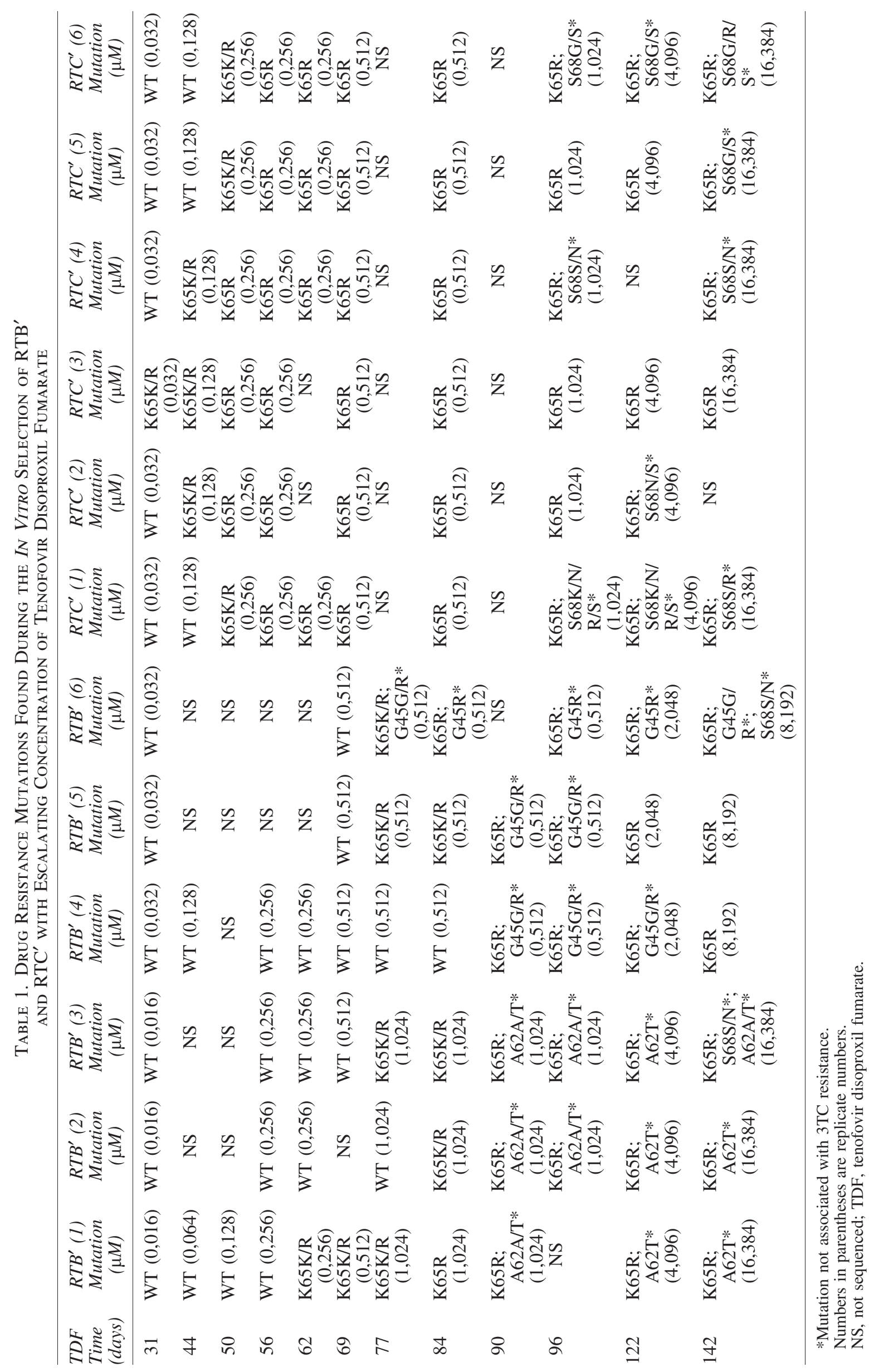


patients infected with subtype $\mathrm{C}$ viruses failing TDFcontaining first-line HAART $(69.7 \%)$ in a cohort from South Africa when compared to data from subtype B-infected patients $(7-15 \%)$ in countries dominated by this variant. ${ }^{20}$

TDF was used in several preexposure prophylaxis studies (PrEP) in mono or dual therapy, and it is highly efficacious in preventing infection in high-risk HIV-infected people. ${ }^{21-23}$ In some of them, when a patient enters the study already infected with HIV-1, there is a risk that K65R will emerge. ${ }^{22,23}$ All studies have been performed in countries in which HIV-1 subtype $\mathrm{C}$ is endemic. Our data suggest that K65R could emerge faster in subtype $C$ viruses than in subtype $B$ viruses in PREP studies when TDF is administered principally as monotherapy. This fact could be a limitation of this approach in countries in which the epidemic is dominated by subtype $\mathrm{C}$ viruses.

Interestingly, secondary mutations (A62T, S68G/N/R, and G45R) were partially or completely acquired for both subtypes after K65R fixation with TDF selection (Table 1). However, mutations in positions 45 or 62 were more common in $\mathrm{RTB}^{\prime}$ viruses and substitutions at position 68 were found more frequently in $\mathrm{RTC}^{\prime}$ viruses (Table 1). This could not be explained by differences in the genetic barrier since the same codon is shared by WT subtype B and C viruses at those positions.

Mutations at positions 62 and 68 have usually been detected in combination with $\mathrm{K} 65 \mathrm{R}$ in patients treated with TDF, and these mutations accumulate after K65R selection. ${ }^{24-26}$ This suggests that mutations at positions 62 and 68 could work as secondary mutations, enhancing TDF resistance together with K65R. However, phenotypic studies did not find any impact of these accessory mutations in TDF resistance levels. ${ }^{24,25}$ Nevertheless, Ross et al., analyzing viral populations in patients failing TDF-containing HAART, found that viral populations with $\mathrm{K} 65 \mathrm{R}+\mathrm{S} 68 \mathrm{~N}$ were more prevalent than populations in which only K65R was selected, suggesting an advantage for viruses carrying both mutations. ${ }^{27}$

In contrast to mutations at amino acids 62 and 68, which were selected only at TDF higher concentrations, mutation G45R emerged at the same time as K65R at low drug concentrations. This mutation is located within a region that contains many RT mutations (M41L, K65R, D67N, insertion at $69, \mathrm{~K} 70 \mathrm{R})$ and could act as a secondary mutation. Surprisingly, little information about G45R exists in the literature. This mutation has also been found in association with $\mathrm{K} 65 \mathrm{R}$ in other in vitro selection experiments with $\mathrm{TDF}^{28}$ However, this mutation has not been explored in TDF-treated patients in conjunction or not with K65R. The prevalence of G45R in the Stanford Database (http://hivdb.stanford.edu/) was $0.1 \%$ for naive or NRTI-only treated individuals. ${ }^{29}$ Further studies to better evaluate this mutation in different subtypes are needed.

To assess whether those secondary mutations in conjunction with K65R would restore the replicative capacity of K65R viruses, MT4 cells were infected in the presence of $0.2 \mu \mathrm{M}$ TDF in triplicate. We observed similar levels of p24 between B K65R viruses and B K65R + A62T or G45R (Fig. $1 \mathrm{D})$ indicating that these mutations were not improving the replicative capacity of subtype $B$ viruses containing the K65R mutation. However, a slight improvement in replicative capacity of viruses carrying K65R was noted in conjunction with S68S/N and S68S/G principally (Fig. 1E).
The kinetics of K65R mutation selection was the same with ddI treatment in our experiments. Similar to what was found in TDF, the K65R mutation was selected faster in subtype $\mathrm{C}$ viruses than in the subtype $\mathrm{B}$ counterpart. The mechanism that explains the faster emergence of K65R in subtype $\mathrm{C}$ viruses when compared to subtype $\mathrm{B}$ isolates has already been elucidated. Although the genetic barrier for $\mathrm{K} 65 \mathrm{R}$ in subtype $\mathrm{B}$ and $\mathrm{C}$ is similar, differences in codon usage in both subtypes occur. Codons 64 (AAA) and 65 (AAG) in subtype $\mathrm{C}$ favor pausing of DNA synthesis by RT yielding a higher mutation rate. ${ }^{30,31}$

This result helps to elucidate the kinetics of K65R selection in HIV-1 subtypes B and C. We need to reiterate that ddI was also used in monotherapy and we do not know if the same DRM profile as well as the accumulation kinetics would occur if combination treatment was utilized in vitro or in vivo. As K65R confers cross-resistance to all NRTIs except AZT, this early emergence in subtype $C$ viruses may impede NRTI ARV changes in second-line therapies since only AZT from NRTIs could be used successfully.

In addition, other known primary resistance mutations were coselected with K65R in subtype C viruses (Table 2). Mutations M184I/V and Q151M were selected in 50\% of RTC $^{\prime}$ virus replicates in conjunction with K65R each. However, even if other primary mutations (M184I or L74V) were partially accumulated at the $\mathrm{RTB}^{\prime}$ virus during in vitro selection with ddI, only K65R was fixed by the end of the experiment (Table 2).

According to the International Aids Society, M184V is not considered a resistance mutation for ddI because there was a reduction in viral load when patients were treated with ddI compared to placebo even when M184V was present. ${ }^{32}$ However, those mutations increased ddI resistance levels 4to 8 -fold in in vitro experiments. ${ }^{33,34}$ Moreover, this mutation confers cross-resistance to other NRTIs such as lamivudine and emtricitabine.

Interestingly, the NRTI multiresistant mutation, Q151M, was selected in three of six RTC' viruses with ddI in our in vitro selection experiments (Table 2 ). This mutation confers resistance to all NRTIs approved by the FDA except TDF. ${ }^{19}$ Q151M and additional secondary mutations, known as compensatory mutations, were initially selected under AZT and/or ddI treatment. ${ }^{35}$ Moreover, Q151M is detected in low frequency in subtype B-infected patients. ${ }^{36}$ However, Q151M was selected in a higher frequency with d4T/ddI treatment in subtype C-infected cohorts. ${ }^{37}$ In addition, Q151M was detected in $11.3 \%$ of patients infected with HIV1 subtype E with $\mathrm{d} 4 \mathrm{~T}$ treatment without ddI. ${ }^{38}$ Our data suggest that ddI in addition to d4T plays an important role in the emergence of Q151M and viral subtype may influence the selection of this important DRM.

In our ddI treatment experiments we detected mutation F77I partially selected together with Q151M (Table 2). Although this mutation localizes at the same position as compensatory mutation F77L, studies should be performed to determine whether F77I could act as a compensatory mutation for Q151M and if there is a subtype C predominance. ${ }^{35}$

One of six replicates of RTB' viruses partially selected L74V initially. However, this mutation could not be fixed and was not detected in further passages. Of note, this was the only replicate of the experiment that did not select K65R, which can be explained by the large impact in replicative 


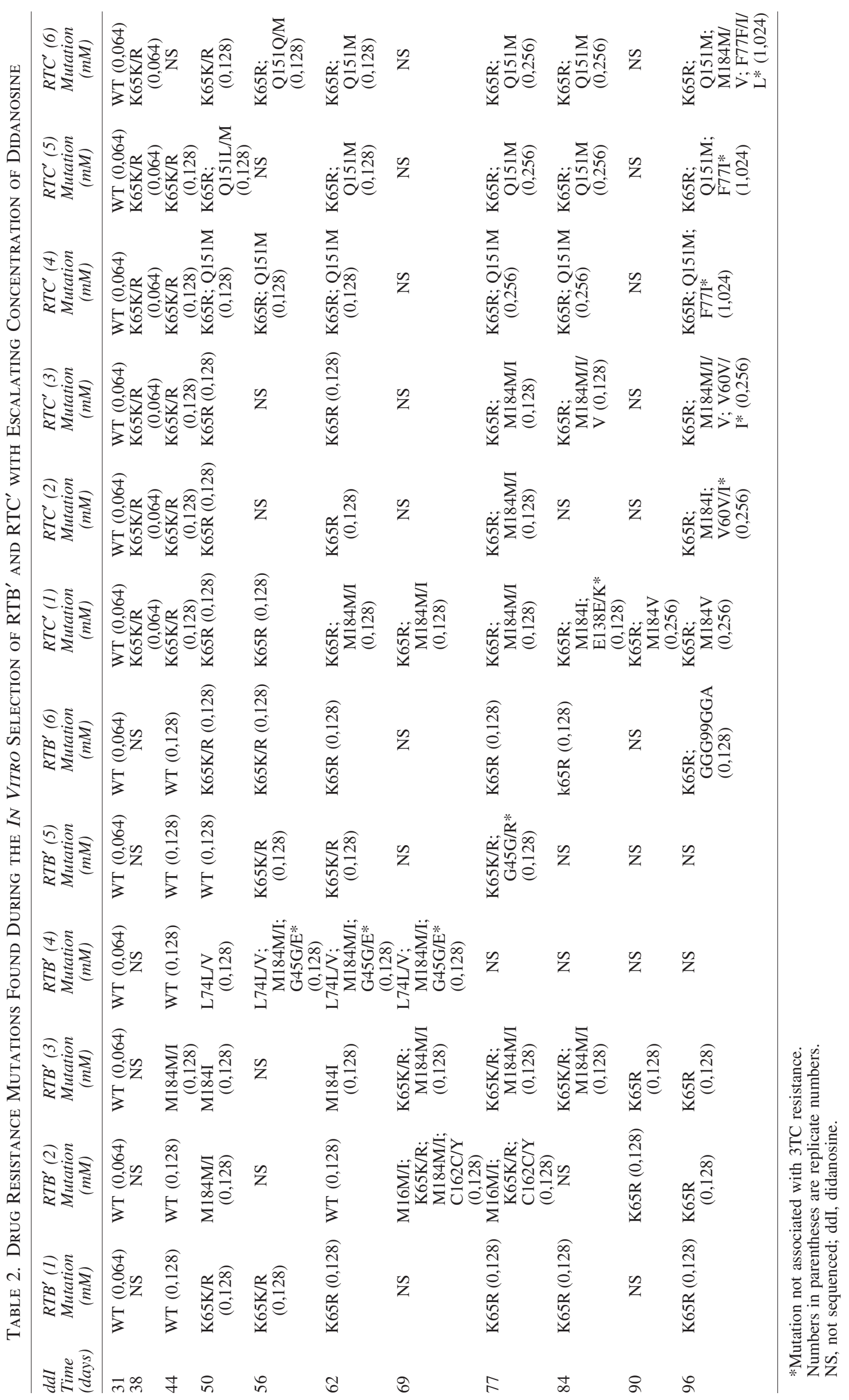


capacity of HIV-1 that happens when L74V and K65R are selected. Perhaps, when it occurs, L74V reverts to WT during virus replication. ${ }^{39,40}$

Some factors could influence the selection of resistance mutations and one of them is genetic background. Rath and colleagues found that genetic background is important for the selection of the nevirapine resistance pathway. However, Rath experiments with two isolates from the same clinical isolate with the same amino acid sequence, and probably the same genetic background, acquired different mutations. Other factors such as epistasis and stochastic effects also contribute to resistance mutations. ${ }^{41}$ Our experiments were performed comparing two genetic backgrounds and it would be interesting to determine whether the same results could be found in viruses $\mathrm{B}$ and $\mathrm{C}$ with different backgrounds, including other molecular clones, and with other cell culture model.

Our results reinforce the previous data on faster selection of K65R in subtype C viruses when compared to subtype B viruses. Independently of the ARV used in our experiments, TDF or ddI, K65R was selected more quickly in $\mathrm{RTC}^{\prime}$ viruses. In addition, we observed different patterns of mutations associated with $\mathrm{K} 65 \mathrm{R}$ in $\mathrm{RTB}^{\prime}$ and $\mathrm{RTC}^{\prime}$ viruses with TDF selection. The actual impact of these findings should be confirmed in patients under HAART regimens composed of TDF.

There was a more complex DRM profile when RTC' viruses were exposed to ddI leading to an accumulation of $\mathrm{K} 65 \mathrm{R}$ and Q151M or K65R and M184I/V. However, only $\mathrm{K} 65 \mathrm{R}$ was accumulated in $\mathrm{RTB}^{\prime}$ viruses. The selection of $\mathrm{Q} 151 \mathrm{M}+\mathrm{K} 65 \mathrm{R}$ and M184V + K65R mutation profiles confers resistance to all FDA approved NRTIs and can represent a great challenge in designing salvage therapies in secondline regimens. These data pose a major concern in using ddI where HIV-1 subtype $\mathrm{C}$ is the major subtype.

We demonstrated the earlier emergence of K65R in subtype C HIV than in subtype B HIV, suggesting that administration of TDF and ddI in subtype $C$ viruses could result in faster therapeutic failure than in subtype B. Antiretroviral treatments need to be well monitored in countries dominated by subtype $\mathrm{C}$ viruses to avoid the accumulation of these complex DRMs.

\section{Acknowledgments}

This work was sponsored by CNPq, FAPERJ, CAPES, and the Brazilian AIDS/STD and Hepatitis Program. We would like to thank Dr. Lucio Gama for reviewing the article.

\section{Author Disclosure Statement}

No competing financial interests exist.

\section{References}

1. (UNAIDS), UJP on H: Global Report: UNAIDS Report on the Global AIDS Epidemic, 2013.

2. WHO: Antiretroviral therapy for HIV infection in adults and adolescents. Recommendations for a public health approach, 2010.

3. Steegen K, Levin L, Ketseoglou I, et al.: High-level crossresistance to didanosine observed in South African children failing an abacavir- or stavudine-based 1st-line regimen. PLoS One 2014;9:e97067.
4. Peters RPH, Van Ramshorst MS, Struthers HE, and McIntyre JA: Clinical assessment of peripheral neuropathy in HIV-infected children on antiretroviral therapy in rural South Africa. Eur J Pediatr 2014;173:1245-1248.

5. Liu H, Ma Y, Su Y, et al.: Emerging trends of HIV drug resistance in Chinese HIV-infected patients receiving firstline highly active antiretroviral therapy: A systematic review and meta-analysis. Clin Infect Dis 2014;59:1495-1502.

6. Lohse N, Hansen A-BE, Pedersen G, et al.: Survival of persons with and without HIV infection in Denmark, 19952005. Ann Intern Med 2007;146:87-95.

7. Hemelaar J, Gouws E, Ghys PD, and Osmanov S: Global trends in molecular epidemiology of HIV-1 during 2000 2007. AIDS 2011;25:679-689.

8. Santos AF and Soares Ma: HIV Genetic diversity and drug resistance. Viruses 2010;2:503-531.

9. Loemba $\mathrm{H}$, Brenner B, Parniak MA, et al.: Genetic divergence of human immunodeficiency virus type 1 Ethiopian clade $\mathrm{C}$ reverse transcriptase (RT) and rapid development of resistance against nonnucleoside inhibitors of RT. Antimicrob Agents Chemother 2002;46:2087-2094.

10. Brenner B, Turner D, Oliveira M, et al.: A V106M mutation in HIV-1 clade $\mathrm{C}$ viruses exposed to efavirenz confers cross-resistance to non-nucleoside reverse transcriptase inhibitors. AIDS 2003;17:F1-F5.

11. Cunha RD, Abreu CM, Gonzalez LMF, et al.: Differential in vitro kinetics of drug resistance mutation acquisition in HIV-1 RT of subtypes B and C. PLoS One 2012;7:e46622.

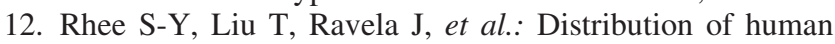
immunodeficiency virus type 1 protease and reverse transcriptase mutation patterns in 4,183 persons undergoing genotypic resistance testing. Antimicrob Agents Chemother 2004;48:3122-3126.

13. Doualla-Bell F, Avalos A, Brenner B, et al.: High prevalence of the K65R mutation in human immunodeficiency virus type 1 subtype $\mathrm{C}$ isolates from infected patients in Botswana treated with didanosine-based regimens. Antimicrob Agents Chemother 2006;50:4182-4185.

14. Brenner BG, Oliveira M, Doualla-Bell F, et al.: HIV-1 subtype $\mathrm{C}$ viruses rapidly develop K65R resistance to tenofovir in cell culture. AIDS 2006;20:F9-F13.

15. Miller MD, Margot N, McColl D, and Cheng AK: K65R development among subtype C HIV-1-infected patients in tenofovir DF clinical trials. AIDS 2007;21:265-266.

16. Von Wyl V, Ehteshami M, Symons J, et al.: Epidemiological and biological evidence for a compensatory effect of connection domain mutation N348I on M184V in HIV-1 reverse transcriptase. $\mathbf{J}$ Infect Dis 2010;201:1054-1062.

17. Van Maarseveen NM, Huigen MCDG, de Jong D, et al.: A novel real-time PCR assay to determine relative replication capacity for HIV-1 protease variants and/or reverse transcriptase variants. J Virol Methods 2006;133:185-194.

18. Iordanskiy S, Waltke M, Feng Y, and Wood C: Subtypeassociated differences in HIV-1 reverse transcription affect the viral replication. Retrovirology 2010;7:1-18.

19. Wensing AM, Calvez V, Günthard HF, et al.: 2014 update of the drug resistance mutations in HIV-1. Top Antivir Med 2014;22:642-650.

20. Sunpath $\mathrm{H}, \mathrm{Wu} \mathrm{B}$, Gordon $\mathrm{M}$, et al.: High rate of K65R for antiretroviral therapy-naive patients with subtype C HIV infection failing a tenofovir-containing first-line regimen. AIDS 2012;26:1679-1684.

21. Karim Q, Karim S, Frohlich J, et al.: Effectiveness and safety of tenofovir gel, an antiretroviral microbicide, for the 
prevention of HIV infection in women. Science 2010;329: $1168-1174$.

22. Thigpen MC, Kebaabetswe PM, Paxton LA, et al.: Antiretroviral preexposure prophylaxis for heterosexual HIV transmission in Botswana. N Engl J Med 2012;367: 423434.

23. Baeten JM, Donnell D, Ndase P, et al.: Antiretroviral prophylaxis for HIV prevention in heterosexual men and women. N Engl J Med 2012;367:399-410.

24. Margot NA, Waters JM, and Miller MD: In vitro human immunodeficiency virus type 1 resistance selections with combinations of tenofovir and emtricitabine or abacavir and lamivudine. Antimicrob Agents Chemother 2006;50: 4087-4095.

25. Stone C, Ait-khaled M, Craig C, et al.: Human immunodeficiency virus type 1 reverse transcriptase mutation selection during in vitro exposure to tenofovir alone or combined with abacavir or lamivudine. Antimicrob Agents Chemother 2004;48:1413-1415.

26. Van Rompay KKA, Johnson JA, Blackwood EJ, et al.: Sequential emergence and clinical implications of viral mutants with $\mathrm{K} 70 \mathrm{E}$ and $\mathrm{K} 65 \mathrm{R}$ mutation in reverse transcriptase during prolonged tenofovir monotherapy in rhesus macaques with chronic RT-SHIV infection. Retrovirology 2007;4:1-22.

27. Ross LL, Rouse E, Gerondelis $\mathrm{P}$, et al.: Low-abundance HIV species and their impact on mutational profiles in patients with virological failure on once-daily abacavir/ lamivudine/zidovudine and tenofovir. J Antimicrob Chemother 2010;65:307-315.

28. Laflamme G, Grant D, White K, et al.: Novel nucleotide inhibitor GS-9148 selects for a K70E mutation in HIV-1 reverse transcriptase and low-level resistance in vitro. Poster Section. Presented at the 47th Interscience Conference Antimicrob Agents Chemother 2007, September. Chicago, IL.

29. Rhee S-Y, Gonzales MJ, Kantor R, et al.: Human immunodeficiency virus reverse transcriptase and protease sequence database. Nucleic Acids Res 2003;31:298-303.

30. Coutsinos D, Invernizzi CF, Xu H, et al.: Template usage is responsible for the preferential acquisition of the K65R reverse transcriptase mutation in subtype $\mathrm{C}$ variants of human immunodeficiency virus type 1. J Virol 2009; 83:2029-2033.

31. Invernizzi CF, Coutsinos D, Oliveira M, et al.: Signature nucleotide polymorphisms at positions 64 and 65 in reverse transcriptase favor the selection of the K65R resistance mutation in HIV-1 subtype C. J Infect Dis 2009;200:12021206.

32. Molina J, Marcelin A-G, Pavie J, et al.: Didanosine in HIV1-infected patients experiencing failure of antiretroviral therapy: A randomized placebo-controlled trial. J Infect Dis 2005;191:840-847.

33. Gu Z, Gao Q, Li X, et al.: Novel mutation in the human immunodeficiency virus type 1 reverse transcriptase gene that encodes cross-resistance to 2', 3'- dideoxyinosine and 2', 3'- dideoxycytidine. J Virol 1992;66:7128-7135.

34. Gao Q, Gu Z, Parniak MA, et al.: The same mutation that encodes low-level human immunodeficiency virus type 1 resistance to $2^{\prime}, 3^{\prime}$-dideoxyinosine and $2^{\prime}, 3^{\prime}$-dideoxycytidine confers high-level resistance to the ( - ) enantiomer of $2^{\prime}, 3^{\prime}$ dideoxy-3'-thiacytidine. Antimicrob Agents Chemother 1993; 37:1390-1392.

35. Shafer RW, Kozal MJ, Winters MA, et al.: Combination therapy with zidovudine and didanosine selects for drugresistant human immunodeficiency virus type 1 strains with unique patterns of pol gene mutations. J Infect Dis 1994; 169:722-729.

36. Waléria-Aleixo A, Martins AN, Arruda MB, et al.: Drug resistance mutation profile and accumulation kinetics in human immunodeficiency virus-positive individuals infected with subtypes $B$ and $F$ failing highly active antiretroviral therapy are influenced by different viral codon usage patterns. Antimicrob Agents Chemother 2008;52: 4497-4502.

37. Kumarasamy N, Madhavan V, Venkatesh KK, et al.: High frequency of clinically significant mutations after first-line generic highly active antiretroviral therapy failure: Implications for second-line options in resource-limited settings. Clin Infect Dis 2009;49:306-309.

38. Nouhin J, Madec Y, Ngo-Giang-Huong N, et al.: Increased risk of Q151M and K65R mutations in patients failing stavudine-containing first-line antiretroviral therapy in Cambodia. PLoS One 2013;8:1-4.

39. Deval J, Navarro J-M, Selmi B, et al.: A loss of viral replicative capacity correlates with altered DNA polymerization kinetics by the human immunodeficiency virus reverse transcriptase bearing the K65R and L74V dideoxynucleoside resistance substitutions. J Biol Chem 2004;279: 25489-25496.

40. Sharma PL, Nurpeisov V, Lee K, et al.: Replicationdependent $65 \mathrm{R}->\mathrm{K}$ reversion in human immunodeficiency virus type 1 reverse transcriptase double mutant K65R + L74V. Virology 2004;321:222-234.

41. Rath Ba, Yousef KP, Katzenstein DK, et al.: In vitro HIV-1 evolution in response to triple reverse transcriptase inhibitors and in silico phenotypic analysis. PLoS One 2013; 8:1-12.

Address correspondence to: Amilcar Tanuri

Laboratório de Virologia Molecular Departamento de Genética Universidade Federal do Rio de Janeiro CCS-Bloco A2, sala 121

Cidade Universitária-Ilha do Fundão 21944-970, Rio de Janeiro-RJ Brazil

E-mail: atanuri@biologia.ufrj.br 